Research Paper

\title{
Donepezil Combined with Natural Hirudin Improves the Clinical Symptoms of Patients with Mild-to-Moderate Alzheimer's Disease: A 20-Week Open-Label Pilot Study
}

\author{
De-qiang Li, Yu-ping Zhou, Han Yang $\bowtie$
}

Department of Integrated Internal Medicine, The First Affiliated Hospital, College of Medicine, Zhejiang University, Hangzhou 310003, Zhejiang, P.R. China.

Corresponding author: Tel: +86-0571-87236809; Email: lucyok@mspil.edu.cn.

(c) Ivyspring International Publisher. This is an open-access article distributed under the terms of the Creative Commons License (http://creativecommons.org/ licenses/by-nc-nd/3.0/). Reproduction is permitted for personal, noncommercial use, provided that the article is in whole, unmodified, and properly cited.

Received: 2012.03.13; Accepted: 2012.05.01; Published: 2012.05.07

\begin{abstract}
Aim: To evaluate the efficacy and safety of donepezil plus natural hirudin in patients with mild-to-moderate Alzheimer's Disease. Methods: In the 20-week, randomized, open-label and controlled study, 84 patients received either donepezil $(5 \mathrm{mg} /$ day for the first 4 weeks and $10 \mathrm{mg} /$ day thereafter) or donepezil plus natural hirudin ( $3 \mathrm{~g} /$ day) treatment. Efficacy was reflected by the change of the total scores of Alzheimer's Disease Assessment Scale cognitive subscale (ADAS-Cog), Activities of Daily Life (ADL) and Neuropsychiatric Inventory (NPI). Results: The patients with the donepezil plus natural hirudin treatment showed more significant improvement in the daily activities and the decline of the cognition than those with donepezil treatment. Significant difference was present in the groups since the 8th week. No group difference was found in the NPI change. However, within the hirudin treatment group, more powerful efficacy including NPI assessment was found in the patients with vascular risk factors (VRF) as comparing to with those without VRF. The combination of donepezil and natural hirudin was well tolerated. The dropout rate was greater in the donepezil and natural hirudin $(50 \%)$ treatment group than in the donepezil (39\%) treatment group. Similar result was found in the incidence of adverse events (23.8\% vs $19.0 \%)$, but there was no statistical difference between the two groups. Adverse events were the most common reason for the dropout. Although hemorrhage and hypersensitiveness were more common in donepezil plus Maixuekang treatment (I $1.9 \%$ and $7.1 \%$ ) group than in donepezil treatment (2.4\% and $2.4 \%)$ group, no significant difference was present between the two groups. Economic problem was another important reason for the patients' withdrawal. Conclusions: Compared with the donepezil treatment in the patients with mild-to-moderate $A D$, our results suggest that donepezil combined with natural hirudin may improve the treatment effects in the ADL, BPSD and cognition of the patients. Furthermore, this joint treatment is safe.
\end{abstract}

Key words: Alzheimer's disease; donepezil; hirudin.

\section{Introduction}

Alzheimer's disease (AD) is characterized clinically by a gradual decline in cognition, continually decreased ability in performing activities of daily living (ADL) and finally increased behavioural and psychological symptoms of dementia (BPSD). At present, there is no etiological cure for AD. Available treatments mainly offer symptomatic benefit such as improving or stabilizing the declined cognition and functional and/or behavioral symptoms. The cholinesterase inhibitor donepezil has been used to treat the 
patients with mild to moderately severe AD in many countries [1]. The studies using the randomized double-blind placebo-controlled trials indicate that donepezil probably potentially benefits the patients with mild to moderate $\mathrm{AD}$ in cognition [2], global function, and activities of daily living [3]. Now the combination of donapezil and other therapeutic strategies like medicamentum alliance is being generally studied in order to get the best treatment outcomes. In China, some traditional herbal medicine combining choline esterase inhibitors is also studied to treat the patients with AD. Thrombosis and ischemia are the commonest vascular risk factors and can result in the occurrence of $\mathrm{AD}$ and aggravated prognosis. Many studies have shown that the improvement of the cerebral blood supply may be helpful in the treatment and rehabilitation of the patients with AD. Typical anticoagulant drugs like heparin are generally used for the prevention and treatment of thrombotic disorders. However, these drugs have certain limitations, including variable anticoagulant effect, the potential to cause bleeding, and an inability to inhibit clot-bound thrombin and thrombocytopenia. These limitations have largely restricted its use in the treatment of AD. Direct thrombin inhibitors, such as hirudin, do not share these limitations. Therefore, hirudin, isolated from the salivary gland of the medicinal leech, is a promising agent and probably replaces heparin in clinic in the future. Natural hirudin plays a prominent role in blood rheology improvement [4] and has been used for many years in China. Here we hypothesize that natural hirudin combined with donepezil may improve the treatment of AD. No report has been published about this combination by now. In this prospective study the efficacy and tolerance of donepezil combined with natural hirudin will be examined on the cognition, ADL and BPSD of the patients with mild to moderate AD.

\section{Methods}

\section{Subjects}

All patients enrolled in this study were diagnosed as possible or probable $\mathrm{AD}$ according to the criteria of National Institute of Neurological and Communicative Disorders and Stroke-Alzheimer's Disease and Related Disorders Association. Subjects with possible cerebrovascular disease such as hypertension and diabetes had to provide eligible evidence that these diseases were controlled. Patients who used memantine or other cholinesterase inhibitors (rivastigmine, galantamine) before but had stopped them for more than 3 months were also allowed because 3 months was an adequate washout period for those drugs based on previous studies [5]. A mini-mental state examination (MMSE) score of 10 26 , and a Hachinski score of $\leq 4$ were used to evaluate the cognitive impairments needed for the diagnosis of AD. Magnetic resonance imaging (MRI) or computed tomography was used to confirm a diagnosis of AD. Other eligible criteria included normal clinical laboratory values and ambulatory status, and sufficient vision and hearing for accomplishing testing procedures. Exclusion criteria included: 1) any active or clinically significant condition affecting the pharmacokinetics of hirudin and donepezil, 2) a known hypersensitivity to hirudin or cholinesterase inhibitors, 3) serious complications of heart, liver or kidney disease, 4) any hematologic disorders, 5) any primary psychiatric disease other than $\mathrm{AD}, 6$ ) focal lesions which were detected by CT or MRI and had clinical significance, 7) delirium complication, and 8) a known or suspected history of alcoholism or drug abuse.

\section{Study design}

In this 20-week, randomized, open-label, controlled study, 103 patients were screened and 84 subjects mentioned their intention to treat at baseline. Patients were randomly assigned to either donepezil (Brand name: Aricept, Eisai Co. Ltd., Japan) treatment or donepezil plus natural hirudin (Brand name: Maixuekang, Guizhou Xinbang Pharmaceutical Co., China) treatment. During the administering phase all participants took Aricept at a dose of $5 \mathrm{mg} /$ day for the first 4 weeks and $10 \mathrm{mg} /$ day thereafter. In the combination treatment, Maixuekang was given at a dose of $3 \mathrm{~g} /$ day for the first 16 weeks and the last 4 weeks was used as its washout period. Patients were required to attend a regular interview every 4 weeks for the following evaluations: routine physical and mental examination, ECG (weeks 8 and 16 only), psychometric tests (ADAS-Cog, ADL and NPI), laboratory assessments (weeks 8 and 16 only), medication compliance check, and adverse events monitoring. At the end, 47 completed the trial. This study was carried out in accordance with the Declaration of Helsinki. Written informed consents were obtained from both the patients and the patient's caregivers.

\section{Assessing measurements}

The Mini-Mental State Examination (MMSE) is widely used to evaluate the cognitive impairments in clinical practice and research. In this study, the basal cognitive function was evaluated by MMSE. The modified Hachinski Ischemic Score was used to detect the vascular cognitive impairment of $\mathrm{AD}$. The Alzheimer's Disease Assessment Scale cognitive subscale (ADAS-Cog) was used to measure the cognitive do- 
mains of AD [6, 7]. Total scores ranged from 0-70. The higher scores indicated greater cognitive impairment. ADL was assessed with a standardized 20-item ADL scale. The scores ranged from 0 to 80 and a higher score indicated less functional ability. The scores were calculated according to the caregiver's description about the patient performance in the first 4 weeks. The Neuropsychiatric Inventory (NPI) was developed to assess the behavioral change in clinical research [8]. The standard NPI evaluated 12 neuropsychiatric disturbances common in dementia and was used in this study to measure the frequency and severity of behavioral psychological symptoms of dementia (BPSD) in the patients. The scores ranged from 0 (no disturbance) to 144 (maximum disturbance).

\section{Data Analyses}

The basal comparison of characteristics in patients with two treatments was analyzed by a chi square test and student's $t$ test. The efficacy was analyzed on the base of the changes from baseline to any time point in total scores of ADAS-Cog, ADL and NPI and the results were shown in figure 2 to figure 4 . For the analysis, a covariance model was used in the treatment group, and clinical time and current dosage were regarded as fixed effects, while baseline score was used as the covariate. This method might reflect a progressive development of $\mathrm{AD}$ and the data would not be missed at random (ie, patients who progress more quickly may be more likely to withdraw). The adverse events and discontinuation rate were analyzed by the Fisher exact test.

\section{Results}

\section{Patient Disposition}

Eighty four patients expressed their intentions to participate in this trial and were divided into the donepezil treatment group and donepezil plus natural hirudin treatment group. The results of the chi square test and student's $t$ test between the two groups did not show any significant difference in demographic data, vascular risk factors, clinical manifestations and neuropsychological measurements. The general characteristics of the two groups were shown in Table 1. All the participants from two groups were also administered the following concomitant medications (donepezil plus Hirudin vs single donepezil treatment): benzodiazepine agents (36.4\% vs $34.8 \%)$, antidepressants $(33.4 \%$ vs $28.4 \%)$, anti hypertensive agents $(27.8 \%$ vs $30.2 \%)$, serum lipid-reducing agents $(20.3 \%$ vs $26.5 \%)$. Statistical analysis showed no significant difference was present $(P>0.05)$, which meant there were no group effect in these most common concomitant medications. 50\% patients in the combined treatment and 39\% patients in the single donepezil treatment did not complete the study due to the following reasons: adverse events $(21.4 \%)$, requests of the patients or career requirement $(9.5 \%)$, admission to nursing at home $(4.8 \%)$, loss in follow-up and other causes (8.3\%). No group differences were found in the reasons led to discontinuation (table 2).

Table I. The characteristics of the patient and the statistical analysis of the results.

\begin{tabular}{|c|c|c|c|}
\hline & Donepezil & Donepezil plus Hirudin & Statistic values \\
\hline Male/female & $17 / 25$ & $20 / 22$ & $x^{2}=0.44 ; P=0.51$ \\
\hline Age (mean $\pm S D)$ & $73.80 \pm 8.17$ & $71.26 \pm 10.25$ & $t=1.26 ; P=0.21$ \\
\hline Education (mean \pm SD) & $9.14 \pm 2.73$ & $8.89 \pm 2.58$ & $t=0.43 ; P=0.67$ \\
\hline Cardiovascular disease (\%) & 23.8 & 26.2 & $x^{2}=0.06 ; P=0.80$ \\
\hline Hypertension (\%) & 38.1 & 31.0 & $x^{2}=0.47 ; P=0.49$ \\
\hline Smoking (\%) & 21.4 & 26.2 & $x^{2}=0.26 ; P=0.61$ \\
\hline Diabetes (\%) & 19.0 & 28.6 & $x^{2}=1.05 ; P=0.31$ \\
\hline Hypercholesterolemia (\%) & 33.3 & 45.2 & $x^{2}=1.25 ; P=0.26$ \\
\hline brain atrophy and/or mild white matter hyperintensity (\%) & 90.5 & 92.9 & $x^{2}=0.16 ; P=0.69$ \\
\hline MMSE score (mean $\pm S D)$ & $20.4 \pm 5.24$ & $19.2 \pm 5.11$ & $t=1.06 ; P=0.29$ \\
\hline ADAS-Cog score (mean \pm SD) & $23.9 \pm 9.7$ & $21.5 \pm 8.8$ & $t=1.19 ; P=0.24$ \\
\hline ADL score (mean \pm SD) & $30.5 \pm 10.1$ & $33.2 \pm 11.4$ & $t=1.15 ; P=0.25$ \\
\hline NPI score (mean \pm SD) & $28.4 \pm 8.6$ & $31.3 \pm 9.1$ & $t=1.50 ; P=0.14$ \\
\hline Duration of $\mathrm{AD}$ (year) (mean $\pm \mathrm{SD})$ & $3.43 \pm 1.08$ & $3.84 \pm 1.11$ & $t=1.72 ; P=0.09$ \\
\hline Comorbidities (\%) & 52.4 & 64.3 & $x^{2}=1.22 ; P=0.27$ \\
\hline Concomitant medications used (\%) & 73.8 & 75.0 & $x^{2}=1.84 ; P=0.18$ \\
\hline
\end{tabular}


Table 2. Reason and frequency of discontinuation in two groups.

\begin{tabular}{|c|c|c|}
\hline & $\begin{array}{l}\text { Donepezi } \\
1\end{array}$ & $\begin{array}{l}\text { Donepezil plus Hiru- } \\
\text { din }\end{array}$ \\
\hline Adverse events & $8(19.0)$ & $10(23.8)$ \\
\hline Reqest of patient or carer & $3(7.1)$ & $5(11.9)$ \\
\hline Access to nursing home & $2(5.8)$ & $2(4.8)$ \\
\hline $\begin{array}{l}\text { Lost to follow up and other } \\
\text { causes }\end{array}$ & $3(7.1)$ & $4(9.5)$ \\
\hline Total & $16(39.0)$ & $22(50.0)$ \\
\hline
\end{tabular}

\section{Efficacy}

The ADAS-Cog scores declined at the beginning of the treatment but increased finally in both groups. This meant more impairment in the cognition of the patients occurred after the treatments. The patients who received donepezil plus Maixuekang demonstrated significant decrease in ADAS-Cog scores and increase in ADL scores. Compared to the donepezil treatment group, the ADAS-Cog scores in the combined group had an obvious decrease $(P<0.05$, univariate Student's $t$ test) and the patients displayed much better in cognition since the 8th week. This result indicated the potency of Maixuekang in delaying the cognitive decline in the patients with $\mathrm{AD}$ as treated with donepezil simultaneously (Fig. 1). The ADL scores significantly improved in donepezil plus Maixuekang treatment group from the third evaluation time point to the end of the study as compared with unique donepezil treatment $(P<0.05$, Fig. 2). BPSD as evaluated by the change of NPI score also improved after the treatment, but no statistical difference was found between the two groups in NPI scores ( $P>0.05$, Fig. 3$)$. In order to further elucidate the benefit of Maixuekang, subjects who participated in the Maixuekang treatment group were continually divided into the group with vascular risk factors (VRF) and the group without VRF. The cognition, daily behaviors and neuropsychological symptoms were compared between the two groups (Fig. 4). Statistical difference was present in ADAS-Cog, ADL and NPI scores from the 4th week to the end. The cognition, daily function and behavioral and psycho- logical symptoms were aggravated as Maixuekang was removed, which further proved the potency of Maixuekang in improving the treatment of $\mathrm{AD}$ and delaying its progress.

\section{Safety}

The most common treatment-related adverse events in this study were nausea, vomiting, headache, anorexia, abnormal dreams, diarrhea, infection, hypertension, bradycardia, accidental injury, hemorrhage and hypersensitiveness (Table 3). Ten participants in the donepezil plus Hirudin group and eight subjects in the single donepezil group experienced mild to moderate some of the adverse events. No serious adverse events or deaths were occurred during the study. The statistical results about the laboratory and auxiliary examination did not show any significance difference. The discontinuation rate due to the adverse events in patients treated with donepezil plus Hirudin $(23.8 \%)$ was similar to that in the donepezil group $(19.0 \%)$. Although the patients in the combining treatment group experienced higher rate of hemorrhage than the patients in the donepezil group $(19.1 \%$ vs $3.3 \%)$, no statistical significance was present $(P=0.08)$.

Table 3. The comparison (Fisher' Exact Test) of the frequency of adverse events in two groups (\%).

\begin{tabular}{llll}
\hline & Donepezil & Donepezil plus Hirudin $P$ values \\
\hline Total adverse events & $30(71.4)$ & $38(90.5)$ & 0.17 \\
Hypersensitiveness & $1(2.4)$ & $3(7.1)$ & 0.24 \\
Hemorrhage & $1(2.4)$ & $5(11.9)$ & 0.08 \\
Bradycardia & $2(4.8)$ & $1(2.4)$ & 0.56 \\
Anorexia & $2(4.8)$ & $2(4.8)$ & 1.00 \\
Hypertension & $2(4.8)$ & $2(4.8)$ & 0.64 \\
Infection & $3(7.1)$ & $3(7.1)$ & 0.67 \\
Abnormal dreams & $2(4.8)$ & $2(4.8)$ & 0.64 \\
Headache & $2(4.8)$ & $3(7.1)$ & 1.00 \\
Vomiting & $3(7.1)$ & $3(7.1)$ & 0.67 \\
Accidental injury & $3(7.1)$ & $4(9.5)$ & 1.00 \\
Nausea & $4(9.5)$ & $4(9.5)$ & 0.70 \\
Diarrhea & $5(11.9)$ & $6(14.3)$ & 1.00 \\
\hline
\end{tabular}




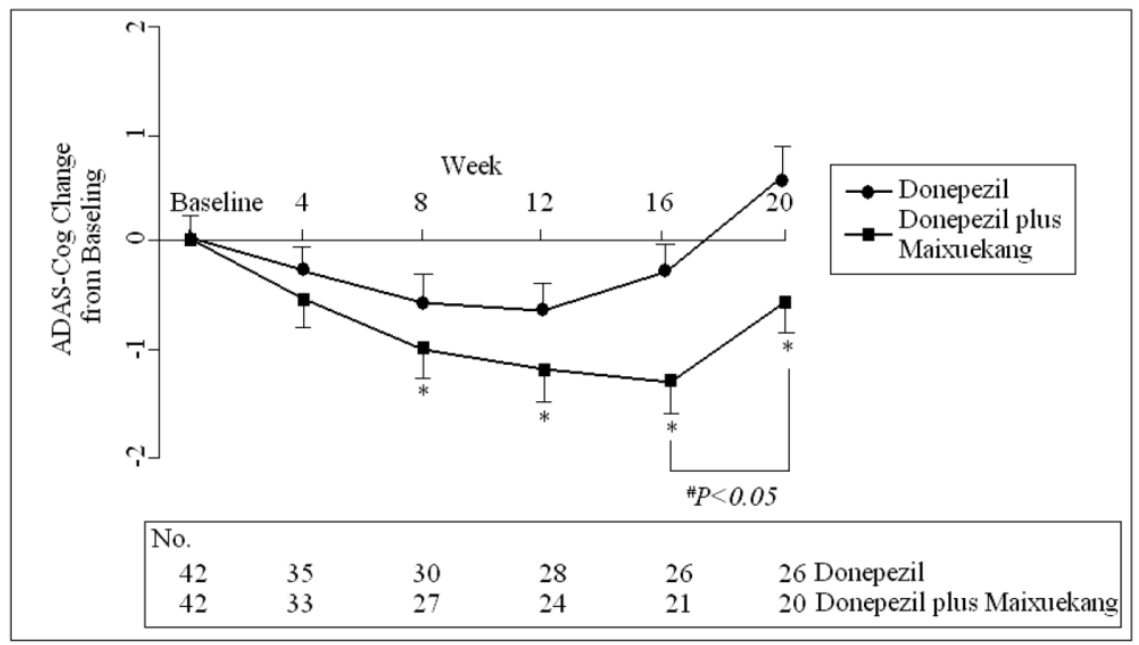

Figure I. The change of the total scores of ADAS-Cog in two groups during the treatment. Data are shown as the means and SD. on the study session for intention-to-treat patients. *: $P<0.05$ between the Donepezil treatment and the Donepezil plus Maixuekang treatment. $\# P<0.05$, comparison between the time point before and after the removal of Maixuekang.

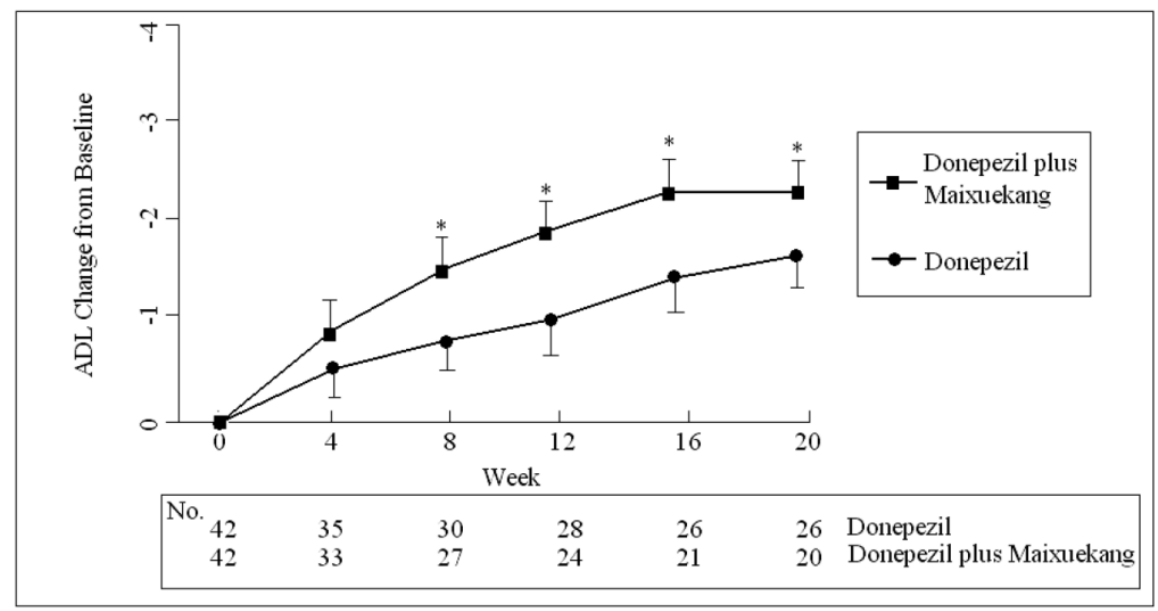

Figure 2. The total score change of ADL total score in two groups during the treatment. Data are shown as the means and SD. *: $P<0.05$, the comparison between the two treatment groups.

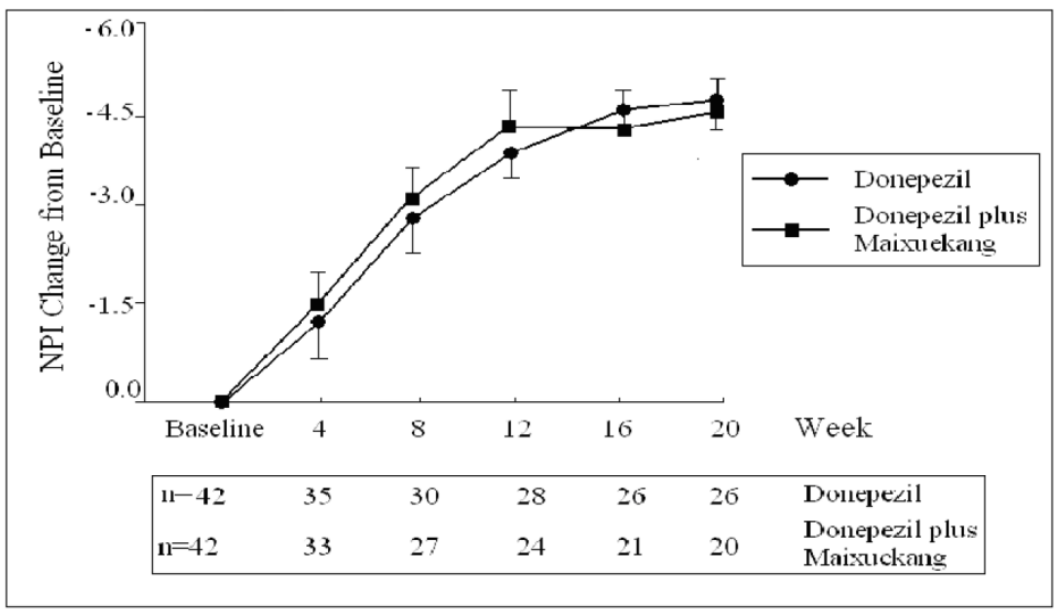

Figure 3. The total score change of NPI in two groups during the treatment. 


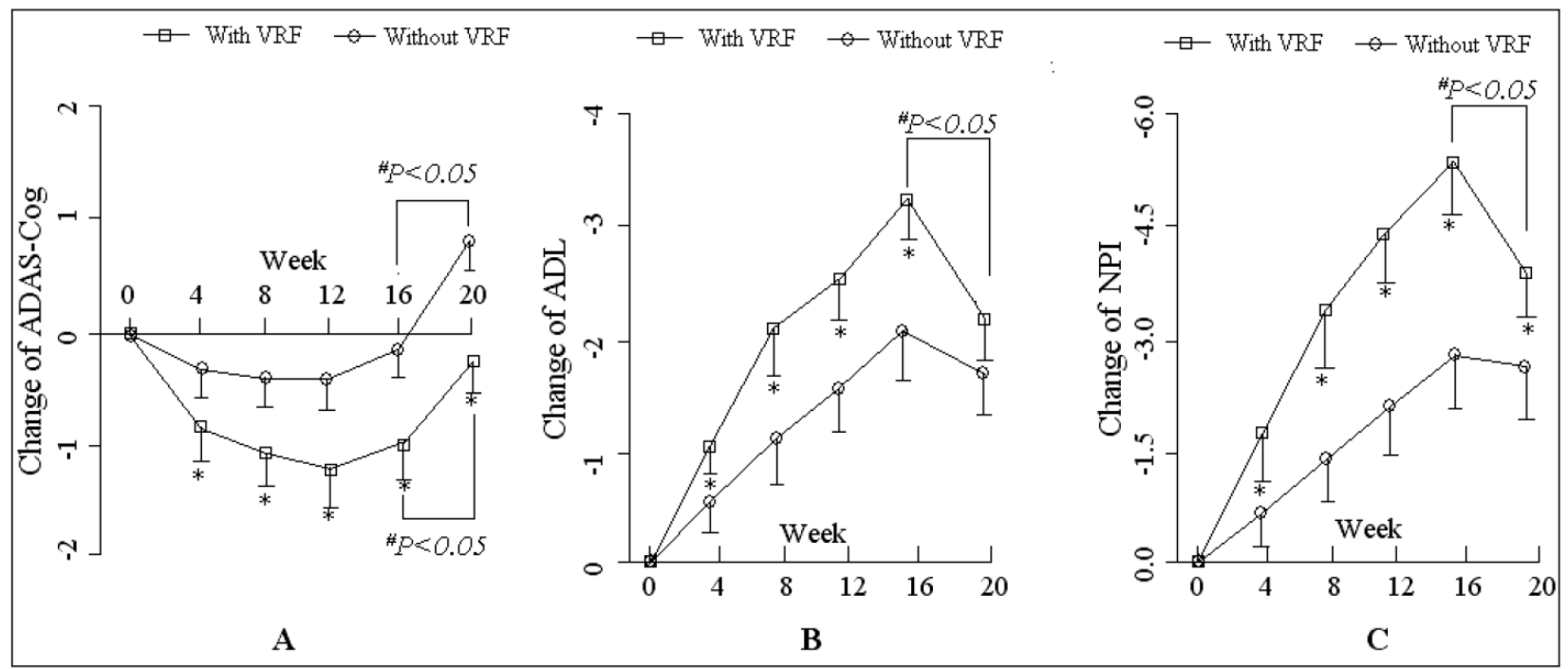

Figure 4. The comparisons of patients with vascular risk factors (VRF and without VRF in the donepezil plus Maixuekang treatment group). The changes of the total scores of ADAS-Cog, ADL and NPI are shown in chart A, B, and C. Data are shown as the means and SD. $* P<0.05$, the comparison between the patients with and without VRF; $\# P<0.05$, the comparison between the time point before and after the removal of Maixuekang.

\section{Discussion}

$\mathrm{AD}$ is a progressive neurodegenerative disease characterized by the loss of cholinergic neurons in the nucleus basalis of Meynert, which is the basement of the cholinergic hypothesis and results in the subsequent development of acetylcholinesterase (AChE) inhibitors in AD treatment [9]. AChE inhibitors are used to treat the patients with mild to moderate AD [10]. Since Donepezil can upregulate AChE activities significantly and increase the protein level of CSF in the patients with $\mathrm{AD}$ [11], it has been widely used in the symptomatic treatments of AD [12]. Many clinical trials have demonstrated that donepezil improves the patients' cognition and some of the BPSD. These improvements of the patients can also reduce caregivers' burden [13]. In this research, after patients with mild to moderate AD took donepezil $5-10 \mathrm{mg} / \mathrm{d}$ for 20 weeks, marked improvements were found in the daily activities, abnormal behaviors and psychiatric symptoms. Although the cognition of the patients was improved shortly at the beginning, it finally inevitably declined. Since no way can stop the progression of AD at the moment [14], the treatments mainly focus on the improvement or stabilization of cognitive, functional and/or behavioral symptoms of the patients. In some cases stabilization based on reducing the worsening speed may be an ideal treatment outcome [15]. In the study, all the evaluation methods did not include the natural progression of $\mathrm{AD}$ after patients were treated by donepezil. This may be a reason why no significant enhancement of cognition is present.
Maybe a larger dose of donepezil instead of a standard dose can give better treatment. Doses of 15 and 20 $\mathrm{mg} /$ day of donepezil have been used in clinic and appeared safe and well tolerated [16]. A recent research reported that a high-dose of donepezil $(23 \mathrm{mg}$ once daily) brought about greater benefits than a standard-dose donepezil ( $10 \mathrm{mg}$ once daily) in patients with moderate to severe $\mathrm{AD}$, but its use was also associated with high rate of withdrawal (30.2\% to $17.9 \%)$ for 24 weeks [17].

In the study, the cognition and daily activities of the patients were improved obviously after Maixuekang was jointly used to the donepezil-treated patients. To further analyze the efficacy of Maixuekang, a comparison was performed between those with VRF and those without VRF. Results showed that the cognition, daily activities and psychiatric symptoms were improved in patients with VRF. In the brain of Alzheimer's patients, the cholinergic neurons innervating the hippocampus and cerebral cortex degenerate before the accumulation of beta-amyloid protein. Donepezil has been testified to protect the cholinergic neurons from the neurodegeneration in olfactory bulbectomy mice [18]. This probably causes the improvement of the cerebral blood flow and the induction of neurogenesis in the hippocampus. Some researchers have already found that at the early stage of dementia a combination of the Abeta oligomers and cerebral ischemia may result in the cholinergic synapse dysfunction and induce memory impairment by cholinergic synapse dysfunction [19]. Abnormal regional brain activity is evident 
in AD patients. This change may be enhanced with synaptic upregulation after a long-term donepezil treatment [20]. It is reported that the cerebral blood flow in the temporal, parietal, and posterior lobes improves significantly after 6 months of the Donepezil treatment (5 mg/day) [21].

Hirudin is a specific thrombin inhibitor. This single-chain polypeptide is composed of 65 amino acid residues and capable to bind thrombin with formation of 1 to 1 non-reversible covalent complexes. Preclinical experiments indicate that it can inhibit thrombosis, decrease the platelet adhesion, reduce the concentration of serum cholesterol and serum triglyceride and improve the cerebral microcirculation. In our study, the improvement of the cognition, daily function and BPSD of the patients in the combined treated group may be due to the improvement of cerebral circulation, reduce of platelet aggregation.

The basic characteristics of patients and the features of the disease ensure the accuracy of the results in this research, for the pharmacotherapy and diagnosis, demographics, medication status, psychometric data and selected clinical and MRI variables may influence the progresses of $\mathrm{AD}$ [22]. Since the severity of the cognitive and functional decline determines the progression of $\mathrm{AD}$ from moderate to severe [23], the neuropsychological assessments measured by ADAS-Cog, ADL and NPI mainly reflect this progress. Of course, as these assessments have certain limitations, they can only contain a certain degree of intelligence dimensions and represent some of the superior brain function.

It is critical for $\mathrm{AD}$ patients to adhere to the medicine treatment. Unfortunately, the medication adherence in these patients is difficult. A big sample survey reported only $58 \%$ of patients were adherent to oral AD medications during the 1-year follow-up period [24]. Once-daily donepezil treatment is a good administration choice for Alzheimer's disease because donepezil can slow the disease progression and improve or stabilize the life quality [25]. However, it is reported that the 1-year persistence rate of donepezil is only $46 \%$ [26]. In this research, the 20 -week dropout rate in the donepezil treatment group is $39 \%$. Possible more patients will drop out with time going on and the rates finally gets close to $46 \% .50 \%$ patients in the donepezil and Maixuekang treatment group discontinue the treatment. This is mainly due to the adverse events and the requirement of the patients and caregivers. The manifestations and frequency of adverse events induced by donepezil in our patients is similar to those reported before [27]. The nature of Maixuekang and its pharmacological effects theoretically determine the high risks of bleeding and allergy, but no statistical difference is present in the frequency of hemorrhage $(11.9 \%$ vs $2.4 \%)$ and hypersensitiveness $(7.1 \%$ vs $2.4 \%)$ between the two groups. This means that Maixuekang can be safely used in the treatment of $\mathrm{AD}$. The most common reason apart from the requirement due to the adverse event for the patients and caregivers who refuse the follow up is the economic load, such as worry about high drug prices $(7.1 \%)$ and lack of medical insurance $(3.6 \%)$. There were no significant differences between the two therapeutic groups $(P>0.05)$. Therefore, improving the patients' economic situation in the future possible better the AD treatment.

However, some limitations are still present in this study. First, these results may partly reflect a normal clinical treatment, but assessors were un-blinded to the time-point of visit. Second, 'ceiling effects' are present, comparing the smaller effect sizes in a limited number of subjects with the broadly defined inclusion criteria for cognitive and behavioral dysfunction. Third, the same instruments are repeatedly used in the interviews and the participants are susceptible to the assessment. A better rigorous research with more patients needs to be performed in the future.

In conclusion, our results show that the symptoms of the AD patients in the donepezil plus natural hirudin treatment group than only treated with donepezil in mild-to-moderate Alzheimer's disease and the formula was safe.

\section{Authors' contributions}

LI De-qiang carried out the follow-up during the treatment, participated in the design of the study and wrote the manuscript. ZHOU Yu-ping did the statistical analysis. HAN Yang conceived the study, and participated in its design and helped to write the manuscript. All authors read and approved the final manuscript.

\section{Competing Interests}

The authors have declared that no competing interest exists.

\section{References}

1. Jones R, Sheehan B, Phillips P, Juszczak E, Adams J, Baldwin A, Ballard C, Banerjee S, Barber B, Bentham P, Brown R, Burns A, Dening T, Findlay D, Gray R, Griffin M, Holmes C, Hughes A, Jacoby R, Johnson T, Jones R, Knapp M, Lindesay J, McKeith I, McShane R, Macharouthu A, O'Brien J, Onions C, Passmore P, Raftery J, Ritchie C, Howard R; DOMINO-AD team: DOMINO-AD protocol: donepezil and memantine in moderate to severe Alzheimer's disease - a multicentre RCT. Trials 2009; 10:57.

2. Inoue J, Hoshino R, Nojima H, Ishida W, Okamoto N. Investigation of responders and non-responders to long-term donepezil treatment. Psychogeriatrics. 2010;10(2):53-61. 
3. Gauthier S, Lopez OL, Waldemar G, Jones RW, Cummings J, Zhang R, Schindler R, Schwam E: Effects of donepezil on activities of daily living: integrated analysis of patient data from studies in mild, moderate and severe Alzheimer's disease. Int Psychogeriatr 2010; 22:973-983.

4. Cornejo-Esquerra A, Talleri-de-Andrea G, de Jesús Blanco-Favela J, Ramos-Mora A, Villarán-Muñoz B: Leech hirudo medicinalis: a therapeutic alternative available in Mexico. Rev Med Inst Mex Seguro Soc 2009;47:335-340.

5. Griffith P, Lichtenberg P, Goldman R, Payne-Parrish J: Safety and efficacy of donepezil in African Americans with mild-to-moderate Alzheimer's disease. J Natl Med Assoc 2006; 98:1590-1597.

6. Michel BF, Luciani V, Geda YE, Sambuchi N, Paban V, Azorin JM. In Alzheimer's disease, the clinical expression of behavioral and psychological signs and symptoms is early and specific of neuropathological stages. Encephale 2010;36(4):314-325.

7. Farlow MR, Cummings JL, Olin JT, Meng X: Effects of oral rivastigmine on cognitive domains in mild-to-moderate Alzheimer's disease. Am J Alzheimers Dis Other Demen 2010;25:347-352.

8. Rocca P, Leotta D, Liffredo C, Mingrone C, Sigaudo M, Capellero B, Rocca G, Simoncini M, Pirfo E, Bogetto F. Neuropsychiatric symptoms underlying caregiver stress and insight in Alzheimer's disease. Dement Geriatr Cogn Disord 2010;30(1):57-63.

9. Spencer JP, Middleton LJ, Davies CH: Investigation into the efficacy of the acetylcholinesterase inhibitor, donepezil, and novel procognitive agents to induce gamma oscillations in rat hippocampal slices. Neuropharmacology 2010; 59(6):437-43.

10. Palmqvist S, Minthon L, Wattmo C, Londos E, Hansson O. A Quick Test of cognitive speed is sensitive in detecting early treatment response in Alzheimer's disease. Alzheimers Res Ther 2010;2(5):29.

11. Hollingworth SA, Byrne GJ: Prescribing trends in cognition enhancing drugs in Australia. Int Psychogeriatr 2010; 30:1-8.

12. Darreh-Shori $\mathrm{T}$, Soininen H: Effects of cholinesterase inhibitors on the activities and protein levels of cholinesterases in the cerebrospinal fluid of patients with Alzheimer's disease: a review of recent clinical studies. Curr Alzheimer Res 2010;7:67-73.

13. Hashimoto M, Yatabe $Y$, Kaneda K, Honda K, Ikeda M: Impact of donepezil hydrochloride on the care burden of family caregivers of patients with Alzheimer's disease. Psychogeriatrics 2009;9:196-203.

14. Fisher A: Cholinergic treatments with emphasis on $\mathrm{m} 1$ muscarinic agonists as potential disease-modifying agents for Alzheimer's disease. Neurotherapeutics 2008;5:433-442.

15. Lu PH, Edland SD, Teng E, Tingus K, Petersen RC, Cummings JL; Alzheimer's Disease Cooperative Study Group: Donepezil delays progression to AD in MCI subjects with depressive symptoms. Neurology 2009;72:2115-2121.

16. Doody RS, Corey-Bloom J, Zhang R, Li H, Ieni J, Schindler R: Safety and tolerability of donepezil at doses up to $20 \mathrm{mg}$ / day: results from a pilot study in patients with Alzheimer's disease. Drugs Aging 2008; 25:163-174.

17. Farlow MR, Salloway S, Tariot PN, Yardley J, Moline ML, Wang Q, Brand-Schieber E, Zou H, Hsu T, Satlin A: Effectiveness and tolerability of high-dose $(23 \mathrm{mg} / \mathrm{d})$ versus standard-dose $(10 \mathrm{mg} / \mathrm{d})$ donepezil in moderate to severe Alzheimer's disease: A 24-week, randomized, double-blind study. Clin Ther 2010;32:1234-1251.

18. Yamamoto Y, Shioda N, Han F, Moriguchi S, Fukunaga K: Donepezil-induced neuroprotection of acetylcholinergic neurons in olfactory bulbectomized mice. Yakugaku Zasshi 2010;130:717-721.

19. Watanabe T, Iwasaki K, Ishikane S, Naitou T, Yoshimitsu Y, Yamagata N, Ozdemir MB, Takasaki K, Egashira N, Mishima K, Fujiwara M: Spatial memory impairment without apoptosis induced by the combination of beta-amyloid oligomers and cerebral ischemia is related to decreased acetylcholine release in rats. J Pharmacol Sci 2008;106:84-91.

20. McGeown WJ, Shanks MF, Forbes-McKay KE, Waiter GD, Elrick I, Venneri MG, Venneri A: Established donepezil treatment modulates task relevant regional brain activation in early Alzheimer's disease. Curr Alzheimer Res 2010;7(5):415-427.

21. Yoshida Y, Sugiyama T, Utsunomiya K, Ogura Y, Ikeda T: A pilot study for the effects of donepezil therapy on cerebral and optic nerve head blood flow, visual field defect in normal-tension glaucoma. J Ocul Pharmacol Ther 2010;26:187-192.
22. Epstein NU, Saykin AJ, Risacher SL, Gao S, Farlow MR; Alzheimer's Disease Neuroimaging Initiative (ADNI): Differences in medication use in the Alzheimer's disease neuroimaging initiative: analysis of baseline characteristics. Drugs Aging 2010;27:677-686.

23. Schwam E, Xu Y: Cognition and function in Alzheimer's disease: identifying the transitions from moderate to severe disease. Dement Geriatr Cogn Disord 2010;29:309-316.

24. Borah B, Sacco P, Zarotsky V: Predictors of adherence among Alzheimer's disease patients receiving oral therapy. Curr Med Res Opin 2010;26:1957-1965.

25. Bassil N, Grossberg GT: Novel regimens and delivery systems in the pharmacological treatment of Alzheimer's disease. CNS Drugs 2009;23:293-307.

26. Herrmann N, Binder C, Dalziel W, Smyth S, Camacho F: Persistence with cholinesterase inhibitor therapy for dementia: an observational administrative health database study. Drugs Aging 2009;26:403-407.

27. Burns A, Gauthier S, Perdomo C: Efficacy and safety of donepezil over 3 years: an open-label, multicentre study in patients with Alzheimer's disease. Int J Geriatr Psychiatry 2007;22:806-812. 